\title{
Technologie en Mécanique
}

\section{Mécanique \& Industries - AFM-Méditerranée - Réseau des Mécaniciens du CNRS}

Les cahiers techniques de la technologie en mécanique constituent un nouveau complément à la revue «Mécanique \& Industries » ouvert aux ingénieurs et techniciens du domaine de la mécanique et technologies connexes, désireux de communiquer leurs savoir-faire, leurs compétences, leurs expériences et leurs réalisations.

Ces cahiers techniques pour publier des savoirfaire, des mises en œuvres technologiques et non des résultats d'essais, offrent un outil de communication ouvert à l'ensemble de la communauté (Universités, Écoles d'ingénieurs, Centres Techniques, Grands Groupes, PME, PMI... ). Ils permettent d'abonder la connaissance technique spécifique, de produire des documents de veille technologique, d'identifier des compétences, et même d'identifier des besoins. Nous veillerons à ce que cette publication ouvre un espace de rencontre des savoirs, offrant ainsi une des clefs pour verrous technologiques.

Ces cahiers techniques sont intégrés à la revue « Mécanique \& Industries » de l'Association Française de Mécanique. Ils apportent un nouvel espace de communication pour les applications des sciences technologiques, répondant ainsi à une demande formulée par la mission ressources et compétences technologiques du CNRS et le réseau des mécaniciens du CNRS.

Le centre régional AFM-Méditerranée a pour mission la coordination des Cahiers Techniques de la Technologie en Mécanique, il associe à cette action le Réseau des Mécaniciens du CNRS. (Décision du Conseil d'Administration de l'AFM, le 2/12/04 et validé en Assemblée Générale du 7/12/04.)

\section{Objectif}

Il s'agit de publier

- des études et réalisations, bureau d'études/atelier.

- des présentations de prototypes, maquettes

- des conceptions de moyens d'essais

$-\ldots$

Dans le but de communiquer

- un savoir-faire transposable à de nouvelles applications dont des applications industrielles

- une synthèse de veille technologique

- une expérience pédagogique remarquable impliquant une entreprise

$-\ldots$

Les propositions sont transmises via la voie hiérarchique et soumises à expertises.

\section{Le comité d'édition}

Le comité d'édition associe les principaux référents de la technologie et de ses applications, il est composé de membres représentant :

Président

Le responsable d'AFM-Méditerranée, centre régional de l'AFM :

Claude REY, UPCAM, UMR CNRS 6181

Les Membres

Représentant le Conseil Scientifique de l'AFM :

Daniel COUTELLIER, LAMIH, ENSIAME, Université de Valenciennes

Alain VAUTRIN, SMS/MEM, École des Mines de SaintÉtienne

Représentant le comité de rédaction de la revue «Mécanique 8 Industries »

Donatien LE HOUEDEC, GeM, École Centrale de Nantes Roger PRUD'HOMME, LMM, UPMC Paris 6

Représentant l'Académie des Technologies

Jean FRENE, LMS, Université de Poitiers

Pierre LAMICQ, ancien Directeur Scientifique et Technologique de la SEP

Représentant l'OSEO-ANVAR

Thien-My PHAN Chargé d'affaires à la Direction de la Technologie, Anvar

Représentant la MRCT (Mission Ressources et Compétences Technologiques UPS 2274 CNRS)

Gérard LELIEVRE, Directeur de la MRCT

Représentant le RDM CNRS (Réseau des Mécaniciens du CNRS)

Bertrand GUILLAUME, responsable du comité de pilotage régional du RDM « Aquitaine »

Richard KOTARBA, responsable du comité de pilotage régional du $\mathrm{RDM}$ « $\mathrm{PACA}$ »

Représentant l'Association des chefs de département GMP des IUT :

Gilles CORNELOUP, Chef du département GMP de l'IUT d'Aix

Abdelaziz HAMZAOUI, Chef du département GMP de l'IUT de Troyes

Représentant le GTT « Transfert et Technologies (fabrication, conception et maintenance) » de l'AFM

Pierre DEVALAN, CETIM Senlis, Président du GTT 
En fonction de leur impact technologique, certaines propositions pourront être proposées en première partie de « Mécanique \& Industries », ou sélectionnées dans l'éventualité d'une aide à valorisation (brevet, enveloppe Soleau...).

\section{Appel à communications}

Les propositions d'articles sont de 6 ou 4 pages au format de publication, et doivent être adressées de préférence par courriel et fichier numérique (Word ou $\mathrm{PDF})$ à

Professeur REY Claude

AFM-Méditerranée/Cahiers Techniques

IMT - La Jetée, bureau 213

38 rue Frédéric Joliot Curie

13451 Marseille Cedex 20

claude.rey@univ.u-3mrs.fr ou

kotarba@L3M.univ-mrs.fr

\section{Diffusion semestrielle spécifique}

Les cahiers techniques de Mécanique et Industrie sont accessibles librement sur le site de l'AFM. Une diffusion semestrielle spécifique supplémentaire, regroupant les articles des cahiers sera faite à l'intention des industries répertoriées par l'AFM et dans les centres de documentations spécialisés dont le CNRS.

Pour le Comité de rédaction de

« Mécanique \& Industries »

Donatien LE HOUEDEC

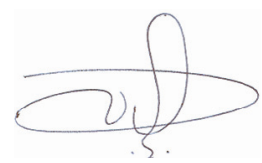

Pour le Comité d'édition

Cahiers Techniques

Claude REY

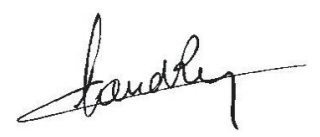

\section{Présentation rapide du Réseau des Mécaniciens du CNRS (RDM)}

Le Réseau des Mécaniciens du CNRS est un réseau thématique national de près de 1000 membres. Il fut créé en 2000 à l'initiative de la Mission des Ressources et Compétences Technologique (MRCT - UPS 2274), qui elle-même au sein de la Direction des Études et Programmes (DEP), met en œuvre la stratégie du CNRS, définie par la Direction Générale, en matière de gestion des ressources et des compétences technologiques utiles aux structures opérationnelles de recherche.

La MRCT compte en son sein 10 réseaux thématiques nationaux (Centres microscopie, Cristaux Massifs, Électrochimie des poudres, Électroniciens, Hautes pressions, Lasers Ultra Rapides, Mécaniciens, Microscopie fluorescence, Opticiens de précision, Plasmas froids), et 5 réseaux thématiques régionaux (Documentalistes, Électroniciens, Mécaniciens, Microscopies).

Le RDM du CNRS se compose d'un Comité de Pilotage de 21 membres (Directoire de 3 membres, 5 chargés d'actions spécifiques, 17 membres actifs représentants les structures régionales). Les structures régionales ont en grande majorité adopté la même représentation, et elles ont pour la plupart d'entre elles associé le Bureau de Formation Permanente de leur délégation régionale à leurs activités.

\section{Objectifs du réseau}

- Offrir un espace d'échanges et de communication :

Rencontres nationales (2000 Satillieu, 2001 Arcachon, 2002 Beaune, 2003 Le Pradet, 2004 Dourdan, 2005 Orléans), Site WEB $\rightarrow$

http://www.cnrs-gif.fr/bfp/sat.html, des listes de discussions, et activité des réseaux régionaux...

- Organiser la régionalisation :

8 régions sont créées soit près de 1000 membres, la $9^{\mathrm{e}}$ et dernière est en cours de création.

- Bilan - État des lieux

Potentiel humain, Parc de machines (Base de Données Nationale et Fiches descriptives des outils et des savoirfaire).

Et mutualiser des outils et des expériences, proposer des formations, diffuser des documents, mettre en place des groupes de travail, ...

Le Réseau des Mécaniciens est adhérant à l'AFM en tant que groupe sectoriel thématique, il participe au GTT « Fabrication, Conception et Maintenance en Mécanique » et contribue au comité d'édition des cahiers techniques. 